\title{
PROPRIEDADES QUÍMICAS E FÍSICAS DE SUBSTRATOS DE ORIGEM MINERAL E ORGÂNICA PARA O CULTIVO DE MUDAS EM RECIPIENTES ${ }^{1}$
}

\author{
PHYSICAL AND CHEMICAL PROPERTIES OF SUBSTRATS \\ WITH MINERAL AND ORGANIC ORIGIN FOR \\ GROWTH OF POTTED PLANTS
}

\author{
José Antônio Kroeff Schmitz ${ }^{2}$ Paulo Vítor Dutra de Souza ${ }^{3}$ Atelene Normann Kämpf ${ }^{4}$
}

RESUMO

Realizou-se a caracterização química e física de cinco materiais [solo, areia, turfa SCv, casca de arroz carbonizada (CAC) e resíduo decomposto de casca de acácia (RDCA)] e de algumas de suas misturas [solo + areia (1:1), solo + areia + CAC (1:1:1), solo + areia + RDCA (1:1:1), turfa SCv + CAC (2:1), turva SCv + RDCA (2:1)] para verificar quais deles podem melhor adequar-se ao uso como substratos para produção de mudas frutíferas e de flores em recipientes. Foram avaliadas as seguintes características: $\mathrm{pH}$ em água $(\mathrm{pH})$, teor total de sais solúveis (TTSS), capacidade de troca de cátions (CTC), teor de carbono orgânico $\left(C_{\text {org. }}\right)$,. densidade seca $(D S)$, porosidade total $(P T)$, espaço de aeração (EA), água disponível (AD), água remanescente $(A R)$ e distribuição do tamanho de partículas. $O$ substrato turfa $S C v+R D C A$ apresentou características químicas e físicas superiores para o uso hortícola.

Palavras-chave: características físicas, características químicas, substrato, turfa, casca de acácia, casca de arroz. carbonizada.

\section{SUMMARY}

Physical and chemical characterizations were done for five materials [soil, sand, peat SCv, carbonized rice husk $(\mathrm{CRH})$ and decomposed acacia (Acacia mearnsii) bark after tannin extraction $(D A B)]$ and some of their mixtures [soil + sand $(1: 1)$, soil + sand + CRH $(1: 1: 1)$, soil + sand $+D A B(1: 1: 1)$, peat $+C R H(2: 1)$, peat $+D A B(2: 1)]$, trying to identify which of them could be used as growing substrates for potted fruit and flower plant crops. The following properties were analyzed: water $\mathrm{pH}$, salinity, cation extange capacity (CEC), organic carbon levels, dry density, total porosity, air space, available water, remaining water and particles distribution. The growing media "peat $S C v+D A B$ " showed upper chemical and physical properties for the horticultural purposes defined.

Key words: physical properties, chemical properties, substrates, peat, carbonized rice husk, acacia bark.

\section{INTRODUÇÃO}

Atualmente, existe uma ampla gama de sistemas de cultivo de mudas frutíferas e de flores em recipientes. Tais sistemas utilizam substratos de origem mineral ou orgânica, natural ou sintética, cujas características diferem marcadamente das do solo (GUERRERO \& POLO, 1989), não existindo um material ou uma mistura de materiais considerada universalmente válida como substrato para todas as espécies (ABAD, 1991). O cultivo em recipientes requer irrigações e fertilizações freqüentes e, para tanto, faz-se necessário o

${ }^{1}$ Extraído da Dissertação de Mestrado do primeiro autor apresentada à Faculdade de Agronomia, Universidade Federal do Rio Grande do Sul (UFRGS), Porto Alegre, RS.

${ }^{2}$ Engenheiro Agrônomo, Mestre em Fitotecnia, Faculdade de Agronomia, UFRGS. Bolsista da CAPES. E-mail: joseschmitz@hotmail.com

${ }^{3}$ Engenheiro Agrônomo, Doutor, Professor Adjunto. Departamento de Horticultura e Silvicultura, Faculdade de Agronomia, UFRGS, CP 776, 91501-970, Porto Alegre, RS. Bolsista do CNPq. E-mail: pvdsouza@ vortex.ufrgs.br. Autor para correspondência.

${ }^{4}$ Biólogo, Doutor, Professor Aposentado, Departamento de Horticultura e Silvicultura, Faculdade de Agronomia., UFRGS. 
conhecimento das propriedades químicas e físicas dos substratos, por serem fatores determinantes no manejo e controle de qualidade dos cultivos.

As propriedades químicas geralmente utilizadas em nível mundial para a caracterização de um substrato são: o $\mathrm{pH}$, a capacidade de troca de cátions (CTC), a salinidade e o teor percentual de matéria orgânica nele presente. Entre as propriedades físicas mais utilizadas, destacam-se: a densidade, a porosidade, o espaço de aeração e a economia hídrica (volumes de água disponíveis em diferentes potenciais). Para cada uma destas propriedades, já foram estudados e definidos padrões e faixas de valores que caracterizam as condições ideais a serem verificadas em um substrato utilizado para produção de mudas de flores e/ou frutíferas em recipientes com irrigação e fertilização ocasionais (BILDERBACK et al., 1982; CONOVER, 1967; BUNT, 1973; DE BOODT \& VERDONCK, 1972; KÄMPF， 2000; PENNINGSFELD， 1983; VERDONCK $\boldsymbol{e t}$ al., 1981; VERDONCK \& GABRIELS, 1988).

No Rio Grande do Sul, a utilização de solo natural (GAULAND, 1997) ou da mistura de solo com areia ainda é prática rotineira dos viveiristas de mudas frutíferas e flores, por sua grande disponibilidade e baixo custo. Porém, estes substratos podem apresentar inconvenientes no crescimento destas plantas, quando utilizados como substrato único, tornando-se necessária a busca de materiais alternativos que permitam melhorar as condições dos substratos utilizados no Estado sem aumentar demasiadamente seu custo.

Existem também, no Estado, indústrias de extração de tanino da casca de Acácia-negra (Acacia mearnsii De Wild.) e engenhos de arroz, geradores de resíduos que podem poluir o ambiente, mas que, por outro lado, são passíveis de serem reciclados. O uso destes resíduos como componentes de substratos hortícolas propicia a obtenção de materiais alternativos, de fácil e constante disponibilidade a baixo custo, auxiliando na minimização da poluição decorrente do acúmulo de resíduos no ambiente.

Outra alternativa regional a ser testada é a turfa, material abundante nos estados do Rio Grande do Sul e Santa Catarina e consagrado internacionalmente por seu uso como componente de substratos hortícolas, sendo utilizado como padrão de comparação no estudo de novos materiais por suas excelentes características físicas.

Este estudo foi realizado para caracterizar alguns exemplares destes materiais acima citados (solo - Argissolo vermelho distrófico arênico; turfa $\mathrm{SCv}$ - Cominas - SC) e algumas de suas misturas em termos químicos e físicos e comparar estes resultados com valores referenciais citados na literatura internacional, para a formulação de substratos para o cultivo de mudas frutíferas e de flores em recipientes.

\section{MATERIAL E MÉTODOS}

Os materiais avaliados foram: solo (horizonte B de um Argissolo vermelho distrófico arênico - PVd), areia (média, de rio), turfa $\mathrm{SCv}$ (turfa vermelha escarificada peneirada com malha de $4,75 \mathrm{~mm}$, oriunda da empresa Cominas, Criciúma - SC), casca de arroz carbonizada (CAC) (mun. de Guaíba, RS, processo de carbonização descrito por BACKES, 1989) e resíduo decomposto de casca de Acácianegra (Acacia mearnsii De Wild.) (RDCA) (resíduo semi-decomposto da extração de tanino peneirado com malha de $4,75 \mathrm{~mm}$, oriundo de depósito da empresa TANAC/SA, Montenegro, RS).

$\mathrm{O} \mathrm{pH}$ em água da turfa $\mathrm{SCv}$ foi previamente avaliado e, devido a seu baixo valor $(3,8)$, foi realizada a determinação de sua necessidade de calagem (BELLÉ, 1990).

As misturas dos materiais foram feitas numa relação de volume por volume, sendo a medição do volume padronizada, conforme método descrito por HOFFMANN (1970), nas seguintes proporções: solo + areia (1:1); solo + areia + CAC $(1: 1: 1)$; solo + areia + RDCA $(1: 1: 1)$; turfa $\mathrm{SCv}+$ CAC (2:1) e turfa SCv + RDCA $(2: 1)$.

As características químicas avaliadas foram: $\mathrm{pH}$ em água $(\mathrm{pH})$, teor total de sais solúveis (TTSS), capacidade de troca de cátions (CTC) e teor de carbono orgânico $\left(\mathrm{C}_{\text {org. }}\right) . \mathrm{O} \mathrm{pH}$ foi determinado através de potenciômetro, em suspensões substrato:água deionizada (1:2,5; volume:volume) (HOFFMANN, 1970). O TTSS foi determinado através de condutivímetro, em suspensão substrato:água deionizada (1:10; peso:volume) (RÖBER \& SCHALLER, 1985). A CTC foi determinada a $\mathrm{pH} \mathrm{7,} \mathrm{com} \mathrm{extratores} \mathrm{tamponados}$ (TEDESCO $\boldsymbol{e}$ t al., 1995). O $\mathrm{C}_{\mathrm{org}}$ foi determinado pelo método Walkley-Black com calor externo (TEDESCO et al., 1995) para os materiais de origem orgânica (turfa SCv, CAC e RDCA). Os teores de carbono dos materiais solo e areia foram avaliados pelo método descrito por TEDESCO et al. (1995) para determinação de porcentagem de MO e posteriormente convertidos para valores de porcentagem de carbono, através do seguinte cálculo: $\% \mathrm{C}_{\text {org. }}=\% \mathrm{MO} / 1,72$.

A determinação das características químicas dos materiais foi realizada no dia 20 de outubro de 1996, em condições de baixa umidade. Já a caracterização das misturas com eles formuladas 
foi realizada 150 dias após a primeira, sendo que as mesmas foram mantidas úmidas ao longo deste período, visando imitar as condições de cultivo. As misturas formuladas com turfa $\mathrm{SCv}$ não foram analisadas para $\mathrm{pH}$, TTSS e CTC, pois os respectivos resultados seriam alterados em função da correção de $\mathrm{pH}$ a ser feita.

Dentre as características físicas, avaliaramse: densidade seca (DS), porosidade total (PT), espaço de aeração (EA), água disponível (AD), água remanescente (AR) e distribuição do tamanho de partículas. Para o cálculo da DS, foi determinado o teor da matéria seca das amostras, através de secagem em estufa a $105^{\circ} \mathrm{C}$ até a estabilização do peso das amostras. A DS foi então determinada pela metodologia proposta por HOFFMANN (1970), através da fórmula:

DS $\left(\mathrm{kg} \mathrm{m}^{-3}\right)=\underline{\text { Dens. úmida }\left(\mathrm{kg} \mathrm{m}^{-3}\right) \times \text { matéria seca }(\%)}$ características químicas foram obtidas a partir de três repetições e as médias das características químicas a partir de duas repetições.

\section{RESULTADOS E DISCUSSÃO}

Considerando que, nos substratos formados por solos de base mineral, a faixa de $\mathrm{pH}$ onde há maior disponibilidade de nutrientes está entre 6 e 7, e que, em substratos de base orgânica, este valor varia, e a faixa ideal está entre 5,2 e 5,5 (KÄMPF, 2000), estas duas faixas de valores foram utilizadas no presente estudo para a comparação entre materiais e misturas de base mineral e orgânica predominante, respectivamente.

Os materiais de base mineral, solo e areia, apresentaram valores de $\mathrm{pH}$ próximo $(5,8)$ ou dentro $(6,3)$ da faixa de $\mathrm{pH}$ ideal para este tipo de material, respectivamente (Tabela 1 ). No entanto, ao contrário
A PT corresponde à umidade presente nas amostras saturadas (tensão 0 $\mathrm{hPa}$; o EA é igual à diferença entre a PT e o volume de água na tensão de $10 \mathrm{hPa}$; a $\mathrm{AD}$ é o volume de água entre $10 \mathrm{e}$ $100 \mathrm{hPa}$, e a AR corresponde ao volume de água remanescente no material após ter sido submetido à tensão de $100 \mathrm{hPa}$. Os valores de PT, EA, $\mathrm{AD}$ e $\mathrm{AR}$ dos materiais e misturas foram determinados a partir de sucções de 0,10 e $100 \mathrm{hPa}$ em funil de tensão (DE BOODT \& VERDONCK, 1972).

A distribuição do tamanho de partículas foi determinada conforme BILDERBACK et al. (1982). Utilizaramse amostras de $100 \mathrm{~g}$ de material seco ao ar. As amostras, colocadas em jogo de peneiras acoplado a agitador mecânico, foram agitadas durante dois minutos a 240 agitações/min. Foram utilizadas peneiras com malhas de 4,76;2,0; 1,0;0,5 e $0,25 \mathrm{~mm}$. As frações retidas em cada peneira foram pesadas e foram calculadas as percentagens sobre o peso total das amostras.

Os resultados foram comparados com os respectivos valores ou faixas de valor considerados ideais pela literatura internacional na formulação de substratos hortícolas. Para fins de comparação entre os materiais, utilizou-se a Análise de Variância e o teste de Duncan a $1 \%$ para comparação das médias dos mesmos. As médias das
Tabela 1 - Características químicas de cinco materiais e cinco misturas com eles formuladas com a finalidade de uso como substratos para produção de mudas em recipientes. Porto Alegre, 1997. N = 3 .

\begin{tabular}{|c|c|c|c|c|c|}
\hline & Substratos & $\begin{array}{c}\mathrm{pH} \\
\left(\mathrm{H}_{2} \mathrm{O}\right)\end{array}$ & $\begin{array}{l}\text { TTSS } \\
\left(\mathrm{g} \cdot \ell^{-1}\right)\end{array}$ & CTC & $\begin{array}{c}\mathrm{C}_{\text {org. }} \\
(\text { ( }(\% \mathrm{MS})\end{array}$ \\
\hline \multirow{5}{*}{ Materiais } & solo & $5,8 c^{1}$ & $0,25 \mathrm{~b}$ & $9,9 \mathrm{c}$ & $1,0 \mathrm{~d}$ \\
\hline & areia & $6,3 \mathrm{a}$ & $0,10 \mathrm{c}$ & $2,1 \mathrm{~d}$ & $0,2 \mathrm{~d}$ \\
\hline & turfa SCv & $3,8 \mathrm{~d}$ & $0,31 \mathrm{~b}$ & $48,7 \mathrm{a}$ & $27,6 \mathrm{~b}$ \\
\hline & CAC & $6,3 \mathrm{a}$ & $0,30 \mathrm{~b}$ & $8,5 \mathrm{c}$ & $17,3 \mathrm{c}$ \\
\hline & RDCA & $6,1 \mathrm{~b}$ & $1,23 \mathrm{a}$ & $38,5 \mathrm{~b}$ & $34,3 \mathrm{a}$ \\
\hline \multirow{5}{*}{ Misturas } & solo + areia $(1: 1)$ & $5,8 \mathrm{a}$ & $0,35 \mathrm{~b}$ & $3,8 \mathrm{~b}$ & $0,8 \mathrm{~b}$ \\
\hline & solo + areia + CAC $(1: 1: 1)$ & 5,9 a & $0,27 \mathrm{~b}$ & $4,2 \mathrm{~b}$ & $1,3 \mathrm{~b}$ \\
\hline & solo + areia + RDCA $(1: 1: 1)$ & $5,4 \mathrm{~b}$ & $0,83 \mathrm{a}$ & $15,5 \mathrm{a}$ & $3,7 \mathrm{~b}$ \\
\hline & turfa SCv + CAC $(2: 1)$ & - & - & - & $24,5 \mathrm{a}$ \\
\hline & turfa SCv + RDCA (2:1) & - & - & - & $27,3 \mathrm{a}$ \\
\hline Ideal $^{2}$ & & $\begin{array}{r}6,0-7,0^{3} \\
5,2-5,5^{4}\end{array}$ & $<0,5$ & $>12$ & $>25$ \\
\hline
\end{tabular}

TTSS: Teor total de sais solúveis; CTC: Capacidade de troca de cátions; $\mathrm{C}_{\mathrm{org}}$ : Carbono orgânico; Turfa SCv: Turfa vermelha Cominas - SC; CAC: Casca de arroz carbonizada; RDCA: Resíduo decomposto de casca de Acácia-negra.

${ }^{1}$ Médias seguidas pela mesma letra não diferem entre si pelo teste de Duncan a $1 \%$. ${ }^{2}$ Valores ideais citados na literatura para os parâmetros avaliados: pH: KÄMPF (2000); TTSS: CONOVER (1967); CTC: PENNINGSFELD (1983); VERDONK et al. (1981) e PENNINGSFELD (1983).

${ }^{3}$ Faixa de $\mathrm{pH}$ ideal para substratos formulados com materiais e misturas de base mineral (KÄMPF, 2000).

${ }^{4}$ Faixa de $\mathrm{pH}$ ideal para substratos formulados com materiais e misturas de base orgânica (KÄMPF, 2000). 
da areia, o solo apresenta uma capacidade de tamponamento de $\mathrm{pH}$ que influencia em sua correção. As misturas formuladas com predominância de materiais de base mineral (solo + areia: 5,8 ; solo + areia + CAC: 5,9 e solo + areia + RDCA: 5,4) apresentaram pH abaixo da faixa ideal. A formulação solo + areia + RDCA apresentou um $\mathrm{pH}$ mais baixo do que as demais misturas e do que o dos seus materiais isolados. Este fato pode ser atribuído à decomposição do material orgânico nele presente, ao longo do período de 150 dias em que as misturas formuladas ficaram sob condições de umidade semelhantes às de cultivo.

A turfa $\mathrm{SCv}$ apresentou o valor de $\mathrm{pH}$ mais baixo $(3,8)$ dentre os materiais de base orgânica analisados (Tabela 1), indicando a necessidade de correção de seu pH para uso como substrato na produção da maioria das mudas em recipientes. Verificou-se que a quantidade de carbonato de cálcio técnico necessária para a correção do $\mathrm{pH}$ desta turfa até 6 (valor estipulado como ideal para as condições de um experimento de cultivo subseqüente, cujos dados não constam neste artigo) corresponderia a 11 quilogramas por metro cúbico.

A CAC e o RDCA apresentaram $\mathrm{pH}$ superior à faixa ideal estipulada, ou seja, 6,3 e 6,1, respectivamente.

A faixa ideal para TTSS encontra-se entre 0 e $0,5 \mathrm{~g} \ell^{-1}$, especialmente para substratos hortícolas utilizados em sistemas de produção nos quais podem ocorrer variações significativas no regime hídrico (CONOVER, 1967). Os materiais solo, areia, turfa $\mathrm{SCv}$ e CAC apresentaram baixos teores de sais (Tabela 1), incluindo-se dentro desta faixa ideal. O material RDCA apresentou um teor de sais $\left(1,23 \mathrm{~g} \ell^{-1}\right)$ superior à faixa ideal de CONOVER, porém é considerado normal dentro da classificação criada por RÖBER \& SCHALLER (1985), podendo ser utilizado em misturas para espécies com média ou alta tolerância à salinidade, segundo PENNINGSFELD (1983).

As misturas solo + areia e solo + areia + CAC apresentaram valores dentro do ideal e solo + areia + RDCA não foi enquadrada dentro da faixa ideal, porém está dentro da faixa tolerável estabelecida por PENNINGSFELD (1983) para plantas sensíveis à salinidade $\left(0,15-1,0 \mathrm{~g} \ell^{-1}\right)$. As misturas à base de turfa $\mathrm{SCv}$ não foram analisadas para TTSS.

Os materiais solo, areia e CAC apresentaram valores de CTC abaixo da faixa ideal estabelecida por PENNINGSFELD (1983) $\left(>12 \mathrm{cmol}_{\mathrm{c}} \ell^{-1}\right)$ para o cultivo de plantas em recipientes, com fornecimento esporádico de nutrientes. Já os materiais turfa SCv1 e RDCA apresentaram valores superiores, sendo, portanto, considerados ideais. Estes valores mais altos são explicados pelo maior teor de carbono orgânico (Tabela 1), e, conseqüentemente, maior teor de matéria orgânica (MO) nestes materiais, já que sua fração húmica é importante fonte de cargas para o substrato, contribuindo assim para o aumento da CTC (ALLISON, 1965). A mistura solo + areia + RDCA atingiu um valor de CTC dentro da faixa ideal, tendo as demais misturas à base de solo + areia ficado abaixo desta faixa. O valor da CTC é importante quando o fornecimento de nutrientes é esporádico, porém torna-se inconveniente quando há fertirrigação contínua.

Considerando o valor mínimo de MO (50\%) estabelecido por VERDONCK $\boldsymbol{e t}$ al. (1981) e PENNINGSFELD (1983), para substratos utilizados na produção de mudas em recipientes, com fornecimento de água e nutrientes esporádico, e assumindo-se que 50 a $60 \%$ da MO é constituída por $\mathrm{C}$, pode-se estabelecer que os teores ideais de carbono orgânico para substratos devem ficar acima de $25 \%$. Segundo este parâmetro, apenas os materiais turfa $\mathrm{SCv}$ e RDCA apresentaram teores suficientes de $\mathrm{C}_{\text {org. }}$ para serem utilizados como componentes únicos de substratos (Tabela 1). A CAC apresentou um valor mais baixo de $\mathrm{C}_{\text {org }}$ $(17,3 \%)$ em relação aos anteriores, devido a seu alto teor de silício e ao processo de carbonização, que sempre implica uma ligeira queima do material original. Os materiais solo e areia foram muito inferiores aos demais quanto à $\mathrm{C}_{\text {org }}$, sendo inadequados como materiais únicos ou combinados entre si para uso como substratos nas condições previamente descritas.

Da mesma forma, as misturas formuladas à base de solo + areia, mesmo com a adição de materiais orgânicos, ficaram muito abaixo do valor mínimo estabelecido como ideal para o conteúdo de carbono orgânico, enquanto aquelas à base de turfa $\mathrm{SCv}$ apresentaram conteúdos em torno do valor preconizado.

A DS ideal para substratos hortícolas está entre 400 e $500 \mathrm{~kg} \cdot \mathrm{m}^{-3}$ (BUNT, 1973). Embora a DS da turfa SCv tenha sido superior ao considerado normal para substratos turfosos por VERDONCK et al. (1981) e WILSON (1983) (45 a 200kg.m ${ }^{-3}$ ), foi inferior àquela considerada ideal (Tabela 2), bem como as dos materiais CAC e RDCA. A característica de baixa densidade desses materiais pode acarretar problemas na fixação das plantas e tombamento, se o cultivo é feito em recipientes altos (JANSEN et al., 1989). No entanto, quando o cultivo é feito em bandejas, necessita-se de substratos leves, pois as baixas densidades não 
comprometem a estabilidade do recipiente. Além disto, as baixas densidades permitem a utilização desses materiais como condicionadores, em misturas com outros materiais de alta densidade. Os materiais solo e areia apresentaram DS muito superior à faixa ideal. Quando empregados sem mistura (s) podem tornar-se inconvenientes pelo peso excessivo que dificulta a manipulação das plantas em recipientes.

A formulação solo + areia aumentou sua DS em relação à dos materiais componentes. Este efeito é esperado em função do ajuste que ocorre entre as partículas e os espaços porosos dos dois materiais (SPOMER,1974). Os materiais orgânicos adicionados a solo + areia reduziram a DS das misturas resultantes, principalmente em solo + areia + CAC, já que CAC apresenta DS muito baixa. No entanto, a proporção formulada $(1: 1: 1)$ não foi suficiente para diminuir a DS das misturas até a faixa ideal. As DS das misturas à base de turfa $\mathrm{SCv}$ mantiveram-se abaixo da faixa ideal, sendo que turfa $\mathrm{SCv}+\mathrm{CAC}$ apresentou uma densidade ainda mais baixa do que a verificada no material turfa $\mathrm{SCv}$, e turfa $\mathrm{SCv}+\mathrm{RDCA}$ ficou com densidade superior a este último. Entretanto, a sugestão de BUNT (1973) para um valor ideal de densidade seca (400$500 \mathrm{~kg} \cdot \mathrm{m}^{-3}$ ) refere-se a cultivos em recipientes plásticos, não havendo, portanto, maiores inconvenientes para a utilização destes substratos para produção de mudas em bandejas.

$\mathrm{O}$ valor ideal da PT para os substratos hortícolas é de $0,85 \mathrm{~m}^{3} \cdot \mathrm{m}^{-3}$ (VERDONCK \&
GABRIELS, 1988). Os materiais CAC, RDCA e turfa $\mathrm{SCv}$ foram os que apresentaram valores de PT mais próximos deste valor ideal, sendo que CAC apresentou o maior volume de poros dentre os materiais analisados (Tabela 2). Os materiais solo e areia apresentaram valores deficientes de PT. A adição de CAC ou RDCA à mistura solo + areia aumentou seu volume de poros, principalmente com a adição de RDCA, não sendo suficiente, no entanto, para garantir uma PT ideal nesta proporção. As misturas à base de turfa $\mathrm{SCv}$ aproximaram-se mais do valor ideal, sendo que turfa $\mathrm{SCv}+\mathrm{RDCA}$ apresentou o melhor resultado para a PT $\left(0,82 \mathrm{~m}^{3} \mathrm{~m}^{-3}\right)$.

$\mathrm{O}$ valor considerado ideal para o EA de substratos hortícolas é de $0,30 \mathrm{~m}^{3} \cdot \mathrm{m}^{-3}$ (PENNINGSFELD, 1983). Este valor se aplica, no entanto, apenas a substratos utilizados em sistemas de produção de mudas em recipientes com irrigação esporádica, pois o volume de ar existente no substrato depende de seu teor e sua capacidade de retenção de água. Os materiais CAC e RDCA apresentaram EAs superiores ao valor considerado ideal, indicando seu uso como condicionadores de substratos como uma boa opção na melhoria do EA destes. A turfa $\mathrm{SCv}$ apresentou um valor muito próximo do ideal, enquanto os materiais solo e areia mostraram-se deficientes para esta característica. A adição de materiais orgânicos à mistura solo + areia elevou seu EA, sendo que as duas misturas resultantes $($ solo + areia + CAC e solo + areia + RDCA) aproximaram-se do valor ideal. Quanto às

Tabela 2 - Características físicas de cinco materiais e cinco misturas com eles formuladas com a finalidade de uso como substratos para produção de mudas em recipientes. Porto Alegre, 1997. $\mathrm{N}=2$.

\begin{tabular}{|c|c|c|c|c|c|c|}
\hline & Substratos & $\begin{array}{c}\text { DS } \\
\left(\mathrm{kg} \mathrm{m}^{-3}\right)\end{array}$ & $\begin{array}{c}\mathrm{PT} \\
\left(\mathrm{m}^{3} \mathrm{~m}^{-3}\right)\end{array}$ & $\begin{array}{c}\text { EA } \\
\left(\mathrm{m}^{3} \mathrm{~m}^{-3}\right)\end{array}$ & $\begin{array}{c}\mathrm{AD} \\
\left(\mathrm{m}^{3} \mathrm{~m}^{-3}\right)\end{array}$ & $\begin{array}{c}\mathrm{AR} \\
\left(\mathrm{m}^{3} \mathrm{~m}^{-3}\right)\end{array}$ \\
\hline Materiais & $\begin{array}{c}\text { solo } \\
\text { areia } \\
\text { turfa } \mathrm{SCV} \\
\mathrm{CAC} \\
\text { RDCA }\end{array}$ & $\begin{array}{l}1.004 \mathrm{~b}^{1} \\
1.335 \mathrm{a} \\
239 \mathrm{c} \\
136 \mathrm{~d} \\
242 \mathrm{c}\end{array}$ & $\begin{array}{l}0,61 \mathrm{c} \\
0,42 \mathrm{~d} \\
0,78 \mathrm{~b} \\
0,85 \mathrm{a} \\
0,79 \mathrm{~b}\end{array}$ & $\begin{array}{l}0,21 \mathrm{~d} \\
0,12 \mathrm{e} \\
0,32 \mathrm{c} \\
0,67 \mathrm{a} \\
0,37 \mathrm{~b}\end{array}$ & $\begin{array}{l}0,20 \mathrm{~b} \\
0,26 \mathrm{a} \\
0,11 \mathrm{~d} \\
0,09 \mathrm{e} \\
0,13 \mathrm{c}\end{array}$ & $\begin{array}{l}0,20 \mathrm{c} \\
0,04 \mathrm{c} \\
0,35 \mathrm{a} \\
0,09 \mathrm{~d} \\
0,29 \mathrm{~b}\end{array}$ \\
\hline Misturas & $\begin{array}{c}\text { solo + areia }(1: 1) \\
\text { solo + areia + CAC }(1: 1: 1) \\
\text { solo + areia + RDCA }(1: 1: 1) \\
\text { turfa SCv + CAC }(2: 1) \\
\text { turfa SCv + RDCA }(2: 1)\end{array}$ & $\begin{array}{l}1.487 \mathrm{a} \\
995 \mathrm{c} \\
1.062 \mathrm{~b} \\
226 \mathrm{e} \\
256 \mathrm{~d}\end{array}$ & $\begin{array}{l}0,41 \mathrm{c} \\
0,58 \mathrm{~b} \\
0,60 \mathrm{~b} \\
0,79 \mathrm{a} \\
0,82 \mathrm{a}\end{array}$ & $\begin{array}{l}0,18 \mathrm{e} \\
0,31 \mathrm{~b} \\
0,27 \mathrm{~d} \\
0,37 \mathrm{a} \\
0,30 \mathrm{c}\end{array}$ & $\begin{array}{c}0,09 \mathrm{c} \\
0,15 \mathrm{ab} \\
0,16 \mathrm{ab} \\
0,14 \mathrm{~b} \\
0,17 \mathrm{a}\end{array}$ & $\begin{array}{l}0,14 \mathrm{~d} \\
0,12 \mathrm{e} \\
0,17 \mathrm{c} \\
0,28 \mathrm{~b} \\
0,35 \mathrm{a}\end{array}$ \\
\hline Ideal $^{2}$ & & $400-500$ & 0,85 & 0,30 & $0,24-0,40$ & $0,25-0,30$ \\
\hline
\end{tabular}

DS = Densidade seca; PT = Porosidade total EA = Espaço de aeração; AD = Água disponível (entre 10 e $100 \mathrm{hPa}) ;$ AR = Água remanescente (acima de 100hPa); Turfa SCv: Turfa vermelha Cominas-SC; CAC: Casca de arroz carbonizada; RDCA: Resíduo decomposto de casca de Acácia-negra.

${ }^{1}$ Médias seguidas pela mesma letra não diferem entre si pelo teste de Duncan a $1 \%$.

${ }^{2}$ Valores ideais citados na literatura para os parâmetros avaliados: DS: BUNT (1973); PT: VERDONCK \& GABRIELS (1988); EA: PENNINGSFELD (1983); AD: DE BOOT \& VERDONCK (1972); AR: VERDONCK \& GABRIELS (1988). 
misturas à base de turfa $\mathrm{SCv}$, houve um acréscimo no EA com a adição de CAC e uma redução com a adição de RDCA. A formulação turfa $\mathrm{SCv}+\mathrm{CAC}$ atingiu um valor alto de EA $\left(0,37 \mathrm{~m}^{3} \cdot \mathrm{m}^{-3}\right)$, superando o valor ideal. Elevados valores de EA podem trazer deficiências hídricas às plantas, especialmente no caso de irrigações pouco freqüentes. Já a mistura turfa $\mathrm{SCv}+\mathrm{RDCA}$ enquadrou-se dentro do valor considerado ideal para o EA $\left(0,30 \mathrm{~m}^{3} \cdot \mathrm{m}^{-3}\right)$.

A faixa de 0,24 a $0,40 \mathrm{~m}^{3} \cdot \mathrm{m}^{-3}$ é aquela considerada ideal para o volume de $\mathrm{AD}$ de substratos utilizados nas condições anteriormente especificadas (cultivo em recipientes) (DE BOODT \& VERDONCK, 1972). A areia apresentou valor de $\mathrm{AD}$ dentro da faixa ideal, o que pressupõe que este material pode garantir elevada disponibilidade de água às plantas. Os materiais $\mathrm{CAC}$, turfa $\mathrm{SCv}$, RDCA e solo apresentaram reduzido volume de AD, ficando sobre ou abaixo do limite mínimo, o que indica que a disponibilidade de água é um fator de restrição de uso destes materiais como substratos únicos dentro das condições acima descritas. O volume de $\mathrm{AD}$ na mistura solo + areia quase foi duplicado pela adição dos materiais orgânicos CAC e RDCA. A adição dos mesmos à turfa $\mathrm{SCv}$ também induziu a um aumento na $\mathrm{AD}$. $\mathrm{O}$ efeito da adição de RDCA superou ao da CAC no sentido de aumentar o volume de água disponível nos substratos. Nenhum substrato formulado atingiu a faixa ideal estabelecida por DE BOOT \& VERDONCK (1972), no entanto, turfa $\mathrm{SCv}+\mathrm{RDCA}\left(0,17 \mathrm{~m}^{3} \cdot \mathrm{m}^{-3}\right)$ foi $\mathrm{o}$ que mais aproximou seu valor desta.

Quanto ao volume de água remanescente, cujo padrão ideal para as condições acima descritas fica na faixa de 0,25 a $0,30 \mathrm{~m}^{3} \cdot \mathrm{m}^{-3}$, segundo VERDONCK \& GABRIELS (1988), o material RDCA foi o único a apresentar valores dentro da mesma. O valor da turfa SCv foi superior ao ideal, podendo este material apresentar problemas por excesso de umidade para as raízes de algumas plantas. Os demais materiais têm AR reduzida. Os materiais areia e CAC destacaram-se por seus volumes de AR muito baixos, característica que demonstra uma deficiência dos mesmos para o cultivo de plantas em recipientes como substratos únicos, em função de sua baixa capacidade de armazenamento de água, condicionando-os a sistemas de irrigação com elevada freqüência. Nenhuma das misturas baseadas em solo + areia atingiu a faixa ideal, embora a adição de RDCA tenha contribuído para aumentar o valor inicial da mistura solo + areia e a adição de CAC tenha diminuído o mesmo. As misturas à base de turfa $\mathrm{SCv}$ mantiveram valores de AR mais elevadas em relação às baseadas em solo + areia, sendo que a adição de $\mathrm{CAC}$ à turfa $\mathrm{SCv}$ induziu a uma redução deste volume de água, permitindo à formulação resultante (turfa $\mathrm{SCv}+\mathrm{CAC}$ ) enquadrar-se dentro da faixa ideal. Isto indica que a adição de CAC à turfa $\mathrm{SCv}$, desde que carbonizada adequadamente, pode diminuir os problemas com excesso de umidade contidos neste material.

Quanto à análise da distribuição do tamanho de partículas, o solo e o RDCA apresentaram partículas com distribuição mais uniforme para todos os tamanhos avaliados, em relação aos demais (Figura 1a). O solo apresentou pequena predominância percentual nos tamanhos intermediários $(2,0-1,0 \mathrm{~mm}$ e $1,0-0,5 \mathrm{~mm})$ e $\mathrm{o}$ RDCA, ausência de partículas maiores do que $4,76 \mathrm{~mm}$. Isso garante a esses materiais uma maior uniformidade de espaços porosos reservados ao ar (especialmente macroporos, no caso do solo), água disponível ( $\mathrm{AD})$ às plantas (poros médios) e água remanescente (AR) (microporos). A areia apresentou alta predominância de partículas de tamanho pequeno $(0,5-0,25 \mathrm{~mm})$ (Figura 1a) e percentagens decrescentes no sentido do aumento do tamanho das partículas. Esta distribuição confere à areia uma grande quantidade de poros médios e grande capacidade de retenção de $\mathrm{AD}$, podendo o EA ficar reduzido por deficiência de macroporos. Na turfa $\mathrm{SCv}$, predominaram as partículas de tamanho grande $(4,76-2,0 \mathrm{~mm})$ com decréscimo percentual no sentido da diminuição do tamanho de partículas (Figura 1a), garantindo ao material um elevado EA e uma elevada AR, em função dos microporos contidos no interior de suas partículas. Porém, a AD desse material é baixa, em função da pequena quantidade de partículas menores do que $1,0 \mathrm{~mm}$. A maior parte das partículas da CAC apresentou tamanho médio (2,0 a $0,5 \mathrm{~mm})$, garantindo alto EA, porém baixa retenção de água.

Resultados semelhantes foram obtidos por BELLÉ (1990) para os materiais areia, turfa e CAC. A mistura solo + areia concentrou sua distribuição nos tamanhos médios a pequenos $(2,0$ - $0,25 \mathrm{~mm})$, apresentando baixos teores percentuais nos extremos (Figura 1b). Esta concentração manteve-se em solo + areia + CAC, porém com predominância de partículas com tamanhos entre 1,0 e $0,5 \mathrm{~mm}$, o que sugere um aumento no EA em relação à mistura anterior. Em solo + areia + RDCA houve uma pequena redução da concentração da distribuição do tamanho de partículas verificada nas misturas anteriores. A adição de materiais orgânicos (CAC e RDCA) à turfa $\mathrm{SCv}$ permitiu uma redução da predominância 


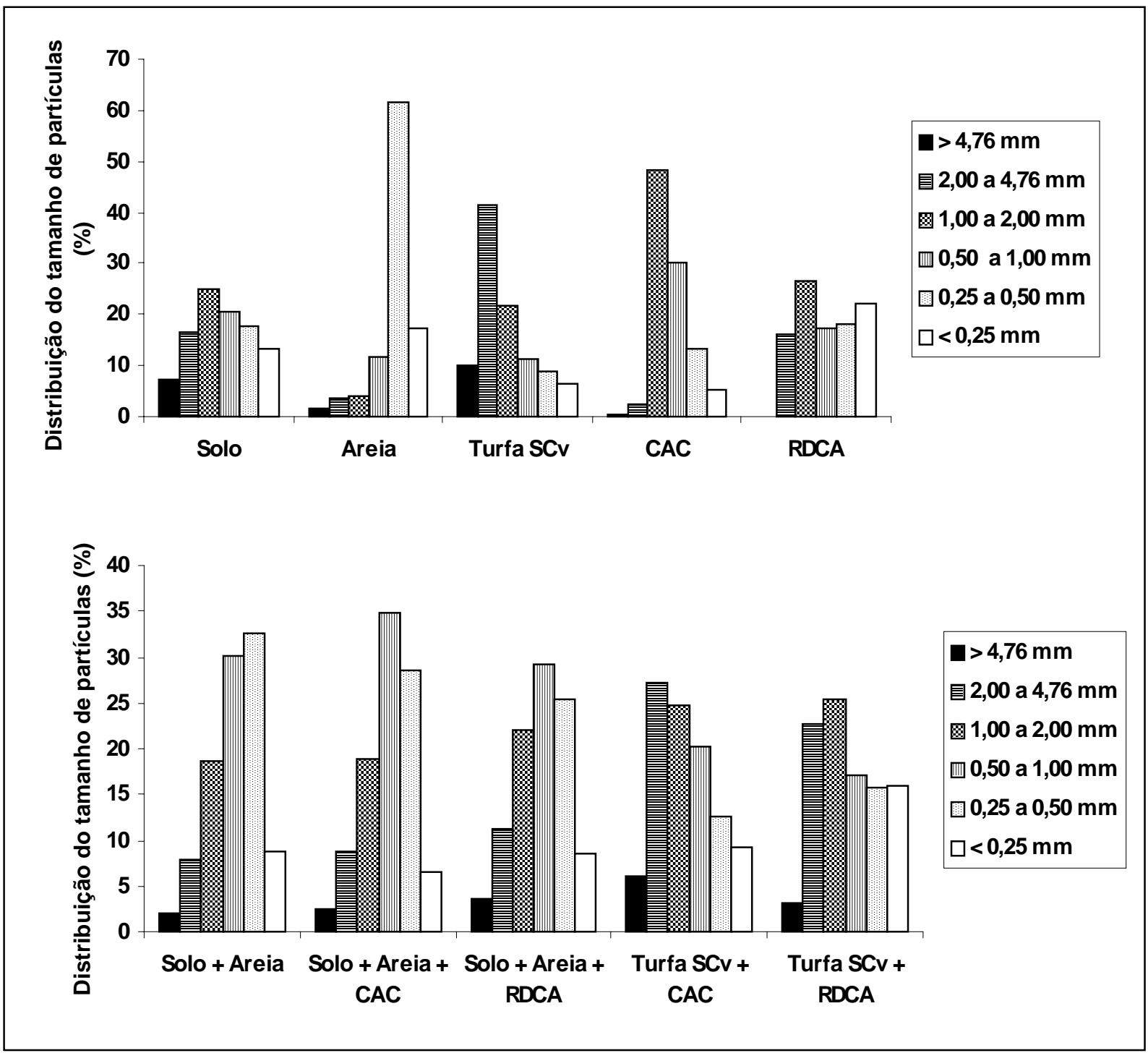

Figura 1 - Distribuição do tamanho de partículas (\%) de cinco materiais (a) e de cinco misturas com eles formuladas (b) com a finalidade de uso como substratos para produção de mudas em recipientes. Porto Alegre, 1997.

de partículas de tamanho grande nela encontrada, especialmente com a adição do RDCA, a qual garantiu maior uniformidade dessa distribuição na formulação resultante, induzindo maior equilíbrio entre EA, AD e AR (Figura 1b).

\section{CONCLUSÕES}

Os materiais e formulações testadas permitem concluir que as mesmas apresentaram propriedades distintas daquelas citadas como ideais para um substrato destinado à produção de mudas frutíferas e de flores em recipientes com irrigação e fertilização esporádicas.

As características que mais se afastaram daquelas ideais foram a capacidade de troca de cátions, os teores de carbono orgânico, a densidade seca e a porosidade total.

A turfa SCv (turfa vermelha escarificada peneirada com malha de $4,75 \mathrm{~mm}$, oriunda da empresa Cominas, Criciúma - SC) é um material promissor como componente de substratos, por suas características químicas e físicas.

A formulação composta por turfa $\mathrm{SCv}+$ RDCA (turfa vermelha escarificada peneirada com malha de $4,75 \mathrm{~mm}$, oriunda da empresa Cominas, Criciúma - SC) (2:1) foi aquela que mostrou características mais favoráveis para o uso almejado.

\section{REFERÊNCIAS BIBLIOGRÁFICAS}

ABAD, M. Los sustratos hortícolas y técnicas de cultivo sin suelo. In: RALLO, L., NUEZ. F. (Eds). La horticultura Española en la C.E.. Reus : Horticultura S.L, 1991. p.271280 .

Ciência Rural, v. 32, n. 6, 2002. 
ALLISON, L.E. Organic carbon. In: BLACK, C.A. Methods of soil analysis. Madison : American Society of Agronomy, 1965. Pt. 2: Chemical and microbiological properties. p.1367-1378.

BACKES, M.A. Composto de lixo urbano como substrato para plantas ornamentais. Porto Alegre, 1989. 78p. Dissertação (Mestrado em Agronomia - Fitotecnia) Programa de Pós-graduação em Agronomia, Universidade Federal do Rio Grande do Sul, Porto Alegre, 1989.

BELLÉ, S. Uso da turfa "Lagoa dos Patos" (Viamão/RS) como substrato hortícola. Porto Alegre, 1990. 143p. Dissertação (Mestrado em Fitotecnia) - Programa de Pósgraduação em Agronomia. Universidade Federal do Rio Grande do Sul, 1990.

BILDERBACK, T.E., FONTENO, W.C., JOHSON, D.R Physical properties of media composed of peanut hulls, pine bark and peatmoss and their effects on azalea growth. Journal of the American Society of Horticultural Science, Alexandria, v.107, n.3, p.522-525, 1982.

BUNT, A.C. Some physical and chemical characteristics of loamless pot-plant substrates and their relation to plant growth. Plant and Soil, The Hague, n.38, p.1954-1965, 1973.

CONOVER, C.A. Soil amendments for pot and field grown flowers. Florida Flower Grower, Florida, v.4, n.4, p.1-4, 1967.

DE BOODT, M., VERDONCK, O. The physical properties of the substrates in horticulture. Wageningen, v.26, p.37-44, 1972.

GAULAND, D.C.S.P. Relações hídricas em substratos à base de turfas sob o uso dos condicionadores casca de arroz carbonizada ou queimada. Porto Alegre, 1997. 107p. Dissertação (Mestrado em Agronomia - Solos) - Programa de Pós-graduação em Agronomia, Universidade Federal do Rio Grande do Sul, Porto Alegre, 1997.
GUERRERO, F., POLO, A. Control de las propriedades hidrofísicas de las turbas para su utilización agrícola. Agr Med, v.119, p.453-459. 1989

HOFFMANN, G. Verbindliche methoden zur untersuchung von TKS und gartnerischen erden. Mitteilungen der VSLUFA, [s.1.], v.6, p.129-153, 1970 .

JANSEN, H., BACHTALER, E., FÖLSTER, E., $\boldsymbol{e}$ t $\boldsymbol{a}$. , Gärtnerischer pflanzenbau. 2. ed. Stuttgart : Ulmer, 1989. $467 \mathrm{p}$.

KÄMPF, A.N. Seleção de materiais para uso como substrato. In: KÄMPF, A.N., FERMINO, M.H. (Eds.) Substrato para plantas: a base da produção vegetal em recipientes. Porto Alegre : Gênesis, 2000. p.139-145.

PENNINGSFELD, F. Kultursubstrate fur den gartenbau, besonders in Deutschland: ein kritischer Überblick. Plant and Soil, The Hague, v.75, p.269-281, 1983.

RÖBER, R., SCHALLER, K. Pflanzenernährung im Gartenbau. Stuttgart : Ulmer, 1985. 352p.

SPOMER, L.A. Two classroom exercices demonstrating the pattern of container soil water distribution. HortScience, Alexandria, v.9, n. 2, p. 152-153, 1974.

TEDESCO, M.J., GIANELlO, C., BISSANI, C.A., et al. . Análises de solo, plantas e outros materiais. 2. ed. Porto Alegre : Departamento de Solos, Faculdade de Agronomia, UFRGS, 1995. 174p. (Boletim Técnico de Solos, 5).

VERDONCK, O., VLEESCHAUMER, D., DE BOODT, M. The influence of the substrate to plant growth. Acta Horticulturae, Wageningen, v.150, p.467-473, 1981.

VERDONCK, O., GABRIELS, R. Substrate requirements for plants. Acta Horticulturae, Wageningen, v.221, p.19-23, 1988.

WILSON, G.C.S. The physico-chemical and physical properties of horticultural substrates. Acta Horticulturae, Wageningen, v.150, p.19-33, 1983 